\title{
Optical characterization of two-dimensional photonic crystals based on spectroscopic ellipsometry with rigorous coupled-wave analysis
}

\author{
Chun-Hung Lin ${ }^{\mathrm{a}, *}$, Hsuen-Li Chen ${ }^{\mathrm{b}}$, Wen-Chi Chao ${ }^{\mathrm{a}}$, \\ Chung-I Hsieh ${ }^{\mathrm{a}}$, Wen-Huei Chang ${ }^{\mathrm{c}}$ \\ a National Nano Device Laboratories, No. 26 Prosperity Road I, Science-based Industrial Park, Hsinchu 300, Taiwan \\ ${ }^{\mathrm{b}}$ Department of Materials Science and Engineering, National Taiwan University, Taipei 106, Taiwan \\ ${ }^{\mathrm{c}}$ School of Applied Chemistry, Chung Shan Medical University, Taichung 402, Taiwan
}

Available online 20 February 2006

\begin{abstract}
A simple and non-destructive optical characterizing method for the 2D photonic crystal (PC) slab was carried out by using specular spectroscopic ellipsometry. The rigorous coupled-wave analysis (RCWA) was further applied to analyze the measured ellipsometric parameters and then to simulate the structure of the measured photonic crystal. A 2D square lattice of silicon rods fabricated by electron-beam lithography on the silicon substrate was used as a testing sample in this study. The reflectance spectrum of the characterized 2D PC was also simulated by RCWA to reflect its photonic bandgap behavior directly.
\end{abstract}

(C) 2006 Elsevier B.V. All rights reserved.

Keywords: Photonic crystal; Rigorous coupled-wave analysis; Ellipsometry

\section{Introduction}

Photonic crystal (PC) [1] with two or three periodic structures has recently received plenty of attention. Spatial refractive index modulation would lead light propagation into definitely different ways as compared with bulk materials. It enables one to manipulate light and then to produce the amazing phenomenon that is impossible with conventional optics. The most important optical property of the PC is its bandgap behaviors. In the bandgap region, there are no corresponding optical states and the incident light is highly reflective. The main difficulties for studying PC are its fabrication and characterization. Although the 3D PC structures can provide much more exciting potential in applications, the fabrication of such PC structures with a photonic bandgap in the visible or infrared regime is a challenge. On the other hand, the 2D PCs have attracted considerable attentions since it would be much more easier to

\footnotetext{
${ }^{*}$ Corresponding author. Tel.: +886 3 5726100/7772; fax: +886 3 5722715.

E-mail address: chlin@mail.ndl.org.tw (C.-H. Lin).
}

fabricate them using the existing semiconductor fabrication methods.

The other problem of the PC development is measurement. The common method in determining the 2D PC structures is to measure the transmittance and reflection spectrum from the PC waveguide [2,3]. However, the coupling loss and the out-of-plane loss may degrade the phenomenon of the PC effects. Previously, a simple and nondestructive optical characterizing method for 2D photonic crystal has been demonstrated by using specular spectroscopic ellipsometry [4]. In this work, the rigorous coupled-wave analysis (RCWA) [5,6] was further applied to analyze the measured ellipsometric parameters and then to simulate the structure and the optical behavior of the measured photonic crystal. A 2D square lattice of silicon rods fabricated by e-beam lithography on the silicon substrate was used as a testing sample in this study.

\section{RCWA analysis}

RCWA is one of the most widely used methods for the rigorous analysis of the optical diffraction by periodic 
gratings. Fig. 1(a) illustrates the geometry of the diffraction configuration for RCWA. The whole structure can be divided into three regions: the incident region (Region I), the grating region (Region II) and the exit region (Region III).

The total electric fields in the external regions (I, III) can be expressed as

$$
\begin{aligned}
& \vec{E}_{\mathrm{I}}=\vec{E}_{\mathrm{inc}}+\sum_{m, n} \vec{R}_{m n} \mathrm{e}^{-\mathrm{j}\left(k_{x m} x+k_{y n} y-k_{\mathrm{I} z, m n n} z\right)} \\
& \vec{E}_{\mathrm{III}}=\sum_{m, n} \vec{T}_{m n} \mathrm{e}^{-\mathrm{j}\left[k_{x m} x+k_{y n} y+k_{\mathrm{III}, m n}(z-h)\right]}
\end{aligned}
$$

where $\vec{E}_{\text {inc }}$ is the incident electric field; $R_{m n}$ and $T_{m n}$ are the electric field amplitudes of $(m, n)$ th reflected and transmitted orders, respectively. The wave vector components $k_{x n}$ and $k_{y m}$ are determined from the Floquet conditions and are given by

$$
\begin{aligned}
& k_{x m}=k_{\text {inc }, x}-m\left(\frac{2 \pi}{\Lambda_{x}}\right) \\
& k_{y n}=k_{\text {inc }, y}-n\left(\frac{2 \pi}{\Lambda_{y}}\right)
\end{aligned}
$$

where $k_{\text {inc }}$ is the wave vector of the incident field; $\Lambda_{x}$ and $\Lambda_{y}$ are the periods of the grating along $x$ and $y$ direction, respectively.

The magnetic fields in the external regions can be obtained from Maxwell's equation

$\vec{H}=-\frac{1}{j \omega \mu_{0}} \nabla \times \vec{E}$ where $\mu_{0}$ is the permeability of free space and $\omega$ is the angular optical frequency.

In the grating region, its periodic permittivity can be expressed in the Fourier expansion

$\varepsilon_{\text {II }}(x, y)=\sum_{g, h} \varepsilon_{g h} \mathrm{e}^{j k_{0}\left(\frac{\lambda_{0} g}{A x} g x+\frac{\lambda_{0}}{A_{y}} h y\right)}$

For a periodic circular pillar shown in Fig. 1(b), the Fourier coefficients $\varepsilon_{g h}$ can be written as [7]

$\varepsilon_{g h}=\left\{\begin{array}{ll}\left(\varepsilon_{2}-\varepsilon_{1}\right) \frac{r}{\rho \Lambda_{x} A_{y}} J_{1}(2 \pi \rho r), & \rho \neq 0 \\ \varepsilon_{1}+\left(\varepsilon_{2}-\varepsilon_{1}\right) \frac{\pi r^{2}}{\Lambda_{x} \Lambda_{y}}, & \rho=0\end{array}, \quad \rho=\sqrt{\left(\frac{g}{\Lambda_{x}}\right)^{2}+\left(\frac{h}{\Lambda_{y}}\right)^{2}}\right.$

The electric and magnetic fields in the grating region can be expressed with a Fourier expansion in terms of the spaceharmonic fields

$\vec{E}_{\mathrm{II}}=\sum_{m, n} \vec{S}_{m n}(z) \mathrm{e}^{-\mathrm{j}\left(k_{x m} x+k_{y n} y\right)}$

$\vec{H}_{\mathrm{II}}=-\mathrm{j} \sqrt{\frac{\varepsilon_{0}}{\mu_{0}}} \sum_{m, n} \vec{U}_{m n}(z) \mathrm{e}^{-\mathrm{j}\left(k_{x m} x+k_{y n} y\right)}$

where $\varepsilon_{0}$ and $\mu_{0}$ are the permittivity and permeability of free space, respectively. $S_{m n}$ and $U_{m n}$ are the amplitudes of the $(m, n)$ th space-harmonic fields.

Finally, by applying the Maxwell's equation in the grating region and matching the boundary conditions at the interface of the three regions, the unknown amplitudes $R_{m n}$ and $T_{n m}$ of the diffracted waves can be determined.

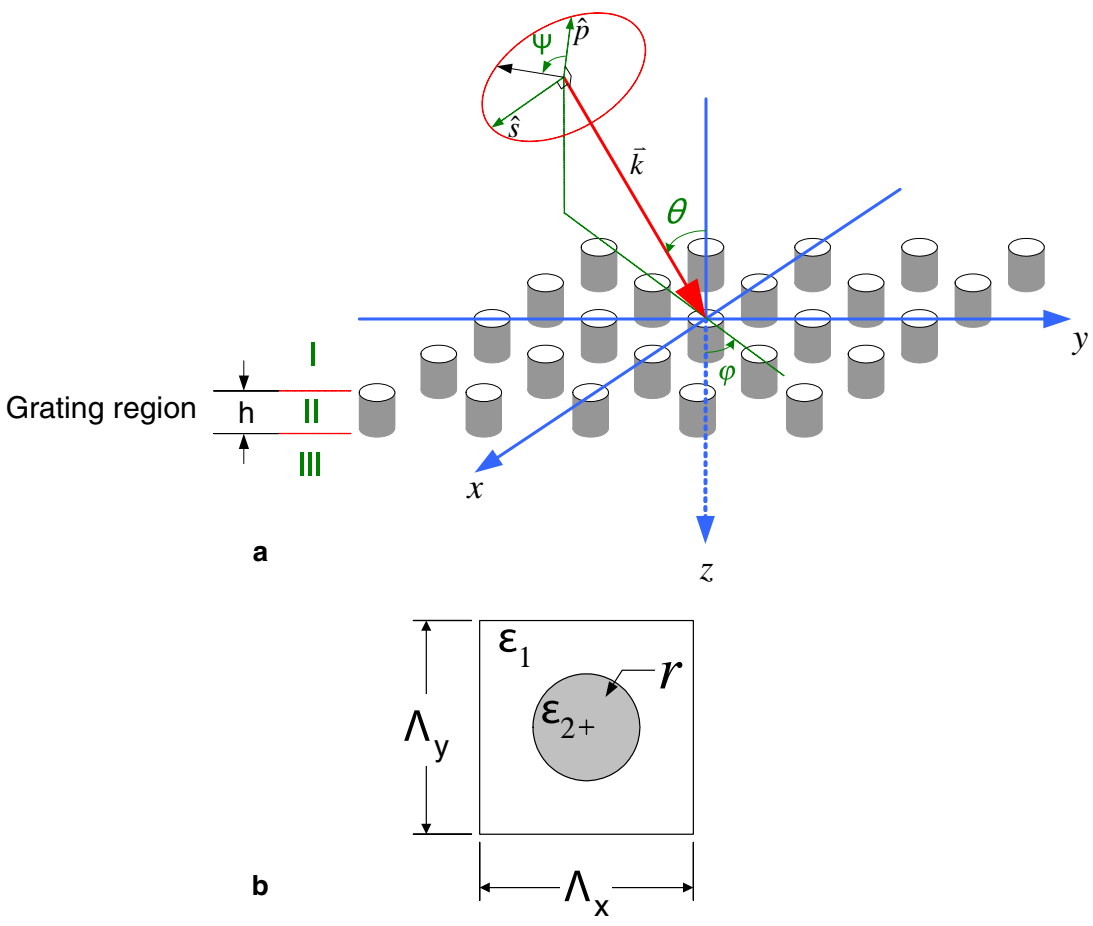

Fig. 1. (a) The geometry of the diffraction configuration for RCWA. (b) Top view of the unit cell of a circular 2D periodic grating. 


\section{Experiment}

\subsection{Fabrication of silicon $2 D$ PC slab}

Fig. 2 shows the fabrication procedure of the 2D PC slab by utilizing the semiconductor process. The substrate is the silicon wafer. To have the enough protection in the subsequent etching, the oxide film with the thickness of $550 \mathrm{~nm}$ was used as the hard mask and was formed on the silicon substrate with a wet oxidation process at $980{ }^{\circ} \mathrm{C}$ in the ASM/LB45 furnace. The $2 \mathrm{D}$ rectangular array with a period of $600 \mathrm{~nm}$ and the rod width of $400 \mathrm{~nm}$ was defined by the electron-beam lithography. The negative tone resist used here was Sumitomo NEB22 with a thickness of $400 \mathrm{~nm}$. The electron-beam patterning was performed on the Leica WEPRINT200 operated at $40 \mathrm{keV}$. After the resist development, the patterns were transferred to the oxide layer by the RIE oxide etcher
(TEL TE5000) and then transferred into the silicon substrate by the poly-silicon etcher (Anelva ECR-6001) with a designed depth of $1 \mu \mathrm{m}$. The ECR microwave power was set to $250 \mathrm{~W}$ and the feed gases were $\mathrm{Cl}_{2}$ with the flow rate of $95 \mathrm{sccm}$ and $\mathrm{O}_{2}$ with the flow rate of $5 \mathrm{sccm}$. Finally, the resist was stripped and the oxide layer was removed by dipping the sample into the HF solution. Fig. 2(b) and (c) show the SEM image of the fabricated 2D PC slab in top view and cross-section view, respectively. The etching depth is around $900 \mathrm{~nm}$. There is a tilt in the sidewall. The top width of the silicon rod is round $360 \mathrm{~nm}$ and the bottom width is round $480 \mathrm{~nm}$.

\subsection{Measurement with spectroscopic ellipsometry}

Fig. 3 shows the experimental configuration of the specular ellipsometric measurement for the PC structure. The measurements were performed on a Sopra SE-5
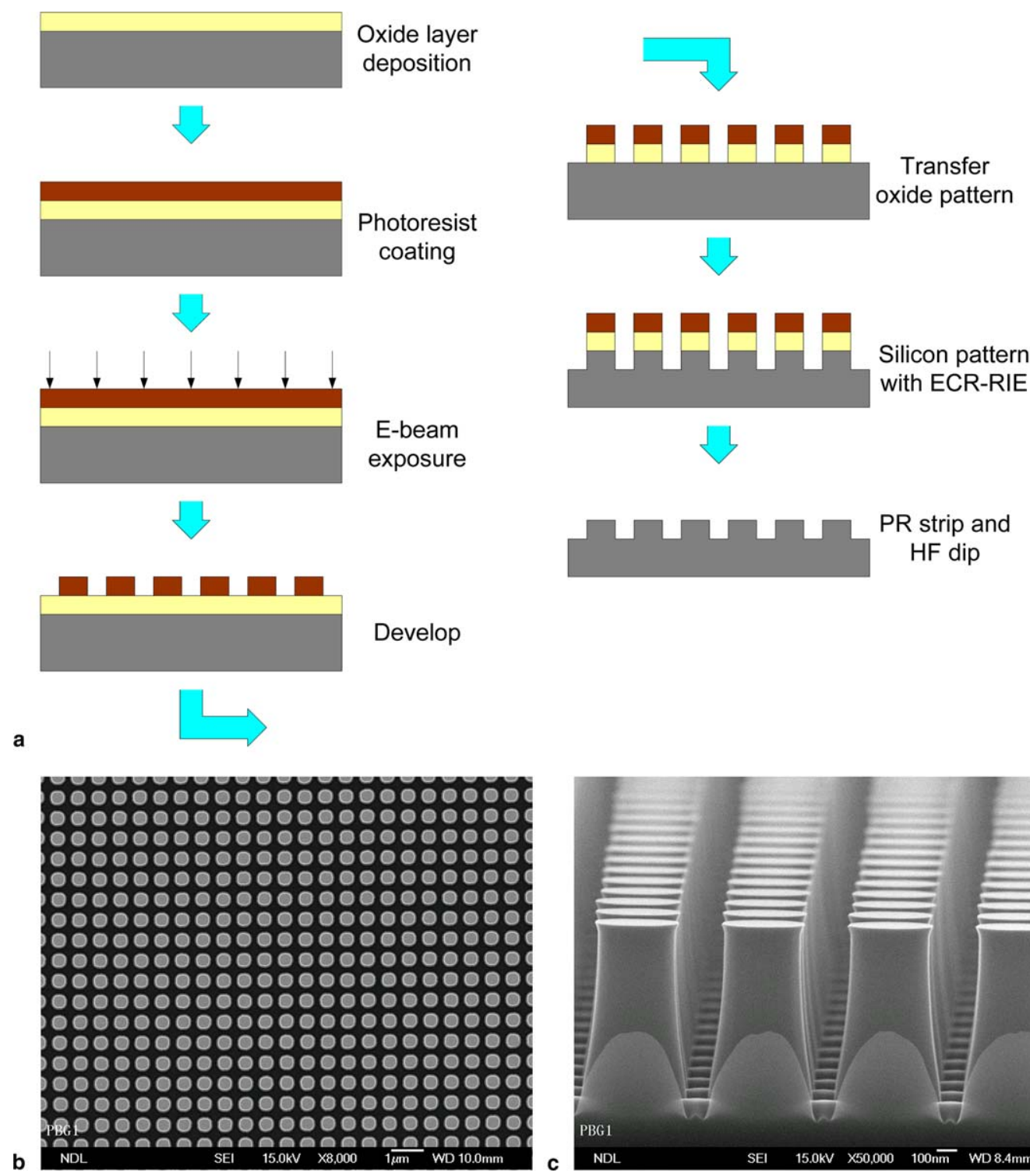


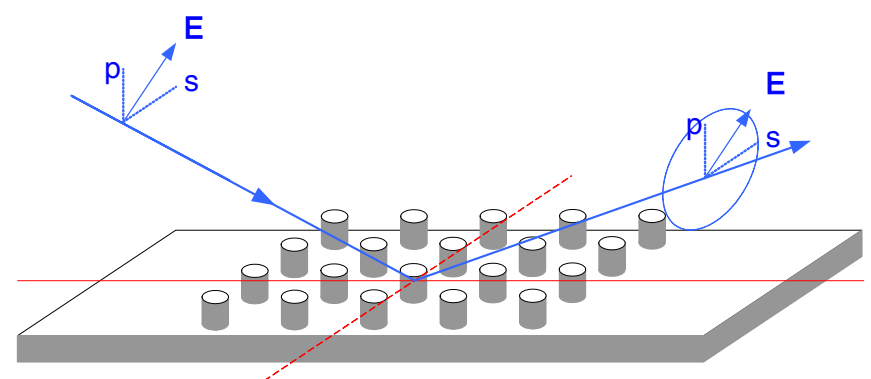

Fig. 3. Schematic diagram of the specular ellipsometric measurement setup for the PC structure.

spectroscopic ellipsometer in the reflection mode. One of the periodic directions of the PC sample was aligned with the plane of the incidence on the ellipsometer. The 0th reflected order of the diffracted waves was measured at the wavelength ranging from 400 to $800 \mathrm{~nm}$. The measured ellipsometric parameters $\tan (\Psi)$ and $\cos (\Delta)$ are defined as

$\rho_{r}=\frac{r_{\mathrm{p}, 0}}{r_{\mathrm{s}, 0}}=\frac{E_{\mathrm{rp}, 0} / E_{\mathrm{ip}, 0}}{E_{\mathrm{rs}, 0} / E_{\mathrm{is}, 0}}=\tan (\Psi) \cdot \mathrm{e}^{\mathrm{i} \Delta}$

where $r_{\mathrm{p}, 0}$ is the 0 th order of the p-polarized reflectance coefficient and $r_{\mathrm{s}, 0}$ is the 0 th order of the s-polarized reflectance coefficient.

\section{Results}

\subsection{Convergency and computing efficiency in $R C W A$}

To perform RCWA, the diffraction orders and the Fourier expansions in the grating region are truncated to a finite number. The accuracy of the analysis depends on the number of retained orders in the calculation. Fig. 4(a) shows calculated ellipsometric parameters of the 0th order of the reflected waves as a function of the highest retained order with respect to the wavelength of 400 and $800 \mathrm{~nm}$, respectively. The parameter $M$ denotes the highest order retained in both of the $x$ and $y$ directions. The convergence rate of RCWA in the $400 \mathrm{~nm}$ wavelength is slower as compared with the calculation in the $800 \mathrm{~nm}$ wavelength. The reason is that more non-evanescent orders are excited by the same grating structure in a shorter wavelength.

To achieve a higher accuracy of RCWA, more retained orders are needed in the calculation. However, the computing time increases exponentially with the increase of the retained orders as shown in Fig. 4(b). Note, the figure is plotted in a log scale. The computing time was measured in the computation of a single wavelength. There is a tremendous difference of the computing time between $1 \mathrm{D}$ and $2 \mathrm{D}$ grating. For example, the computing time of $2 \mathrm{D}$ grating was 3447 times than that of $1 \mathrm{D}$ grating when $M=8$ and was 18,684 times when $M=12$. The major computing time is consumed in solving the eigenvalue problem of the coupled set first order differential equation. For a given highest retained order $M$ in a $1 \mathrm{D}$ grating, the retained orders are $0, \pm 1, \pm 2, \ldots, \pm M$. The matrix size of
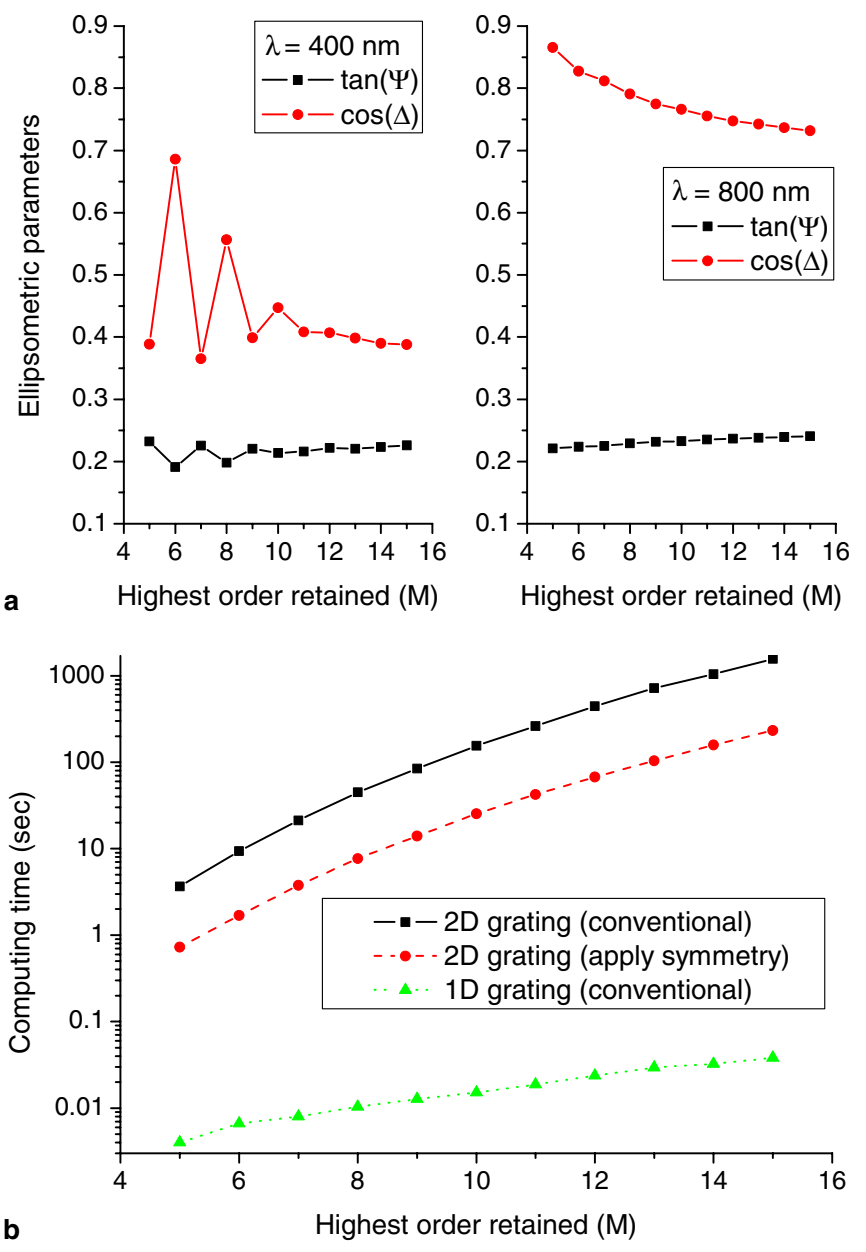

Fig. 4. (a) The calculated ellipsometric parameters of the 0th order of the reflected waves as a function of the highest retained order with respect to the wavelength of 400 and $800 \mathrm{~nm}$, respectively. (b) The computing time as a function of the highest retained order for 1D and 2D gratings.

the eigenvalue problem is $2(2 M+1) \times 2(2 M+1)$. However, for a $2 \mathrm{D}$ grating, the matrix size is $2(2 M+1)^{2} \times 2(2 M+1)^{2}$, which is much more larger. Therefore, there should be a trade-off between the accuracy and the computational expense.

The RCWA for the 2D grating is demanding of memory and time-consuming. To achieve a reasonable computing efficiency, the symmetric condition of 2D grating was further considered and implemented in our RCWA simulation [8]. As shown in Fig. 4(b), the computing time for the new algorithm was reduced by factors of 5.86 for $M=8$ and 6.56 for $M=12$, respectively. The computation was performed on a workstation with the AMD Opteron 844 CPU operating at $1.8 \mathrm{GHz}$.

\section{2. $R C W A$ of $2 D$ photonic crystal}

In the following RCWA simulation, the highest retained order $M$ was chosen to be 8 as a trade-off between the accuracy and the computing time if not specially mentioned. To simplify the computation, the silicon rods were assumed to be the prefect cylinders with the period of $600 \mathrm{~nm}$ so that 
only a single grating layer was considered in the simulation. Two unknown geometrical parameters were needed to determine, the width $w$ and the height $h$ of the silicon rod. Fig. 5 shows the dependencies of the ellipsometric spectra over the rod radius and height. It is found that the ellipsometric spectra are sensitive on the grating geometry. Fig. 6 shows the measured and simulated ellipsometric spectra in various incident angles ranging from $70^{\circ}$ to $85^{\circ}$. The rod width of $396 \mathrm{~nm}$ and the rod height of $900 \mathrm{~nm}$ were used in the simulation. The phase term, $\cos (\Delta)$, was found to be a better parameter as used to characterize the PC structure. There is a difference in $\tan (\Psi)$ term between measured and simulated spectra. However, the shapes of measured and simulated $\tan (\Psi)$ spectra are similar. In addition, the ellipsometric spectra at the incident angle of $70^{\circ}$ were calculated with the highest retained order $M=12$. As compared with the spectra of $M=8$, the results are similar except for the wavelength smaller than $470 \mathrm{~nm}$. This confirms again with the results from Fig. 4(a) that RCWA in shorter wavelength has a slower convergence rate.

With an extension to the multi-layer analysis, RCWA is applicable to 3D PC structures. In addition, the 2D periodic structure with an arbitrary profile can be approxi-
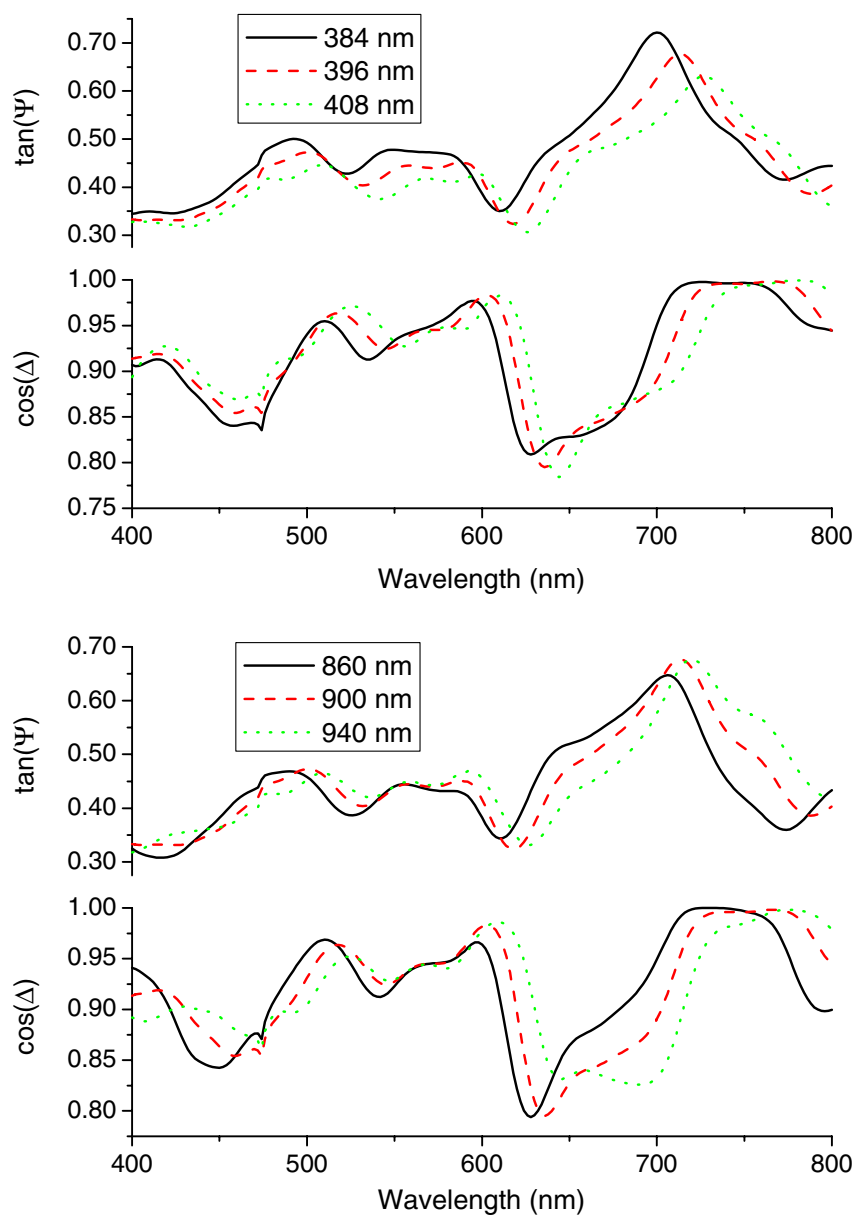

Fig. 5. Simulation of the ellipsometric spectra over: (a) the width $w$ and (b) the height $h$ of the silicon rod. The incident angle is $75^{\circ}$.
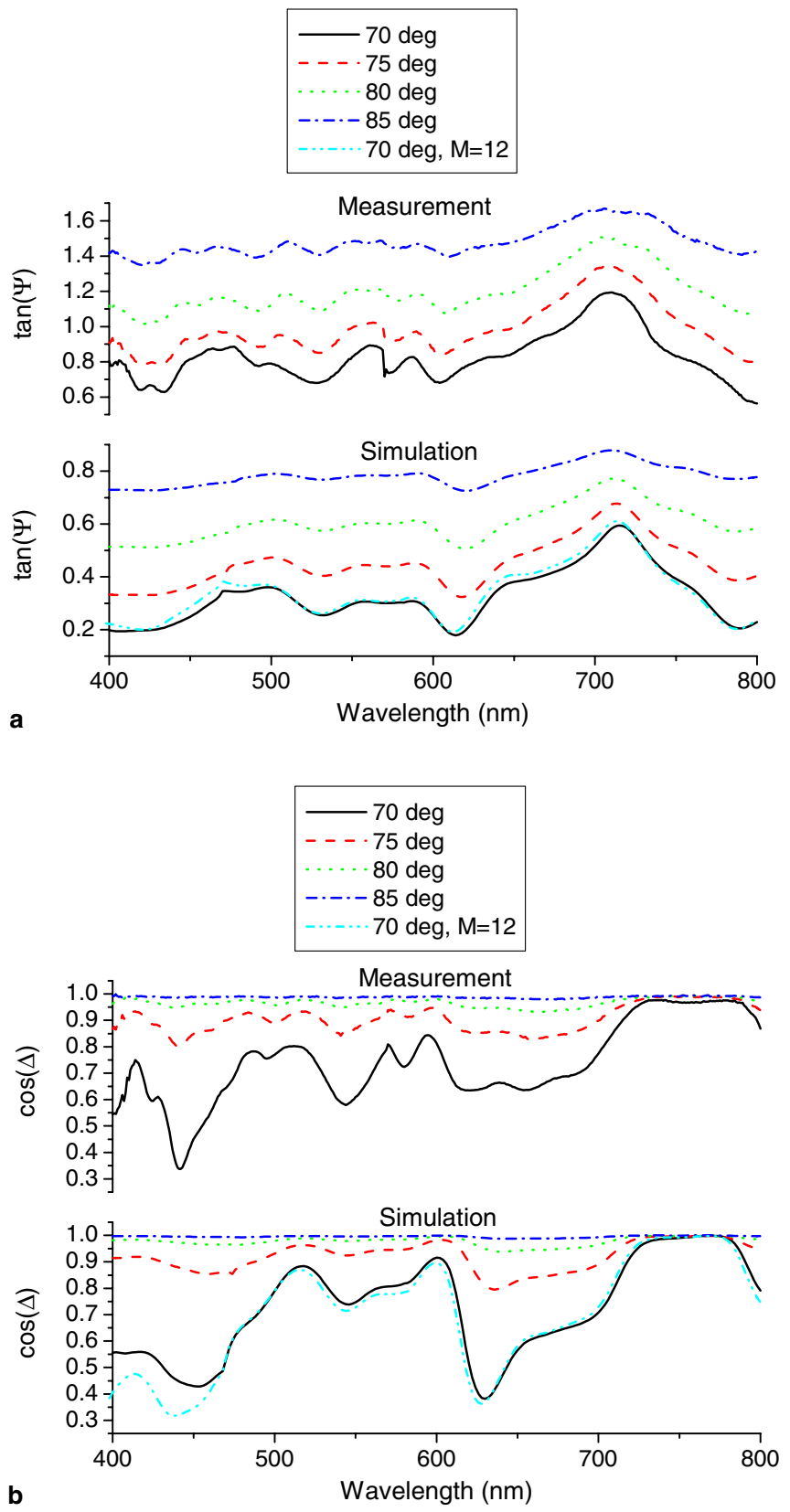

Fig. 6. Measured and simulated specular ellipsometric spectra: (a) $\tan (\Psi)$ and (b) $\cos (\Delta)$ of the fabricated $2 \mathrm{D}$ PC slab. The rod width $=396 \mathrm{~nm}$, height $=900 \mathrm{~nm}$ in the simulation.

mated as a stack of lamellar gratings in RCWA as shown in Fig. 7(a). To have a better approximation with the experimental result, we further considered the circular silicon rod with a trapezoidal profile in its cross-section. The continuous trapezoidal profile was approximated by fivelayer 2D grating with an equal thickness. The top width and the bottom width of the silicon rod are $360 \mathrm{~nm}$ and $432 \mathrm{~nm}$, respectively. The height of the rod is $900 \mathrm{~nm}$. The highest retained order is 12 in this analysis. As shown in Fig. 7(b), the simulated spectra have a better agreement with the experimental result. But there is still room to improve it. A complicated profile with the multi-layer approximation is the way. However, there will be many 

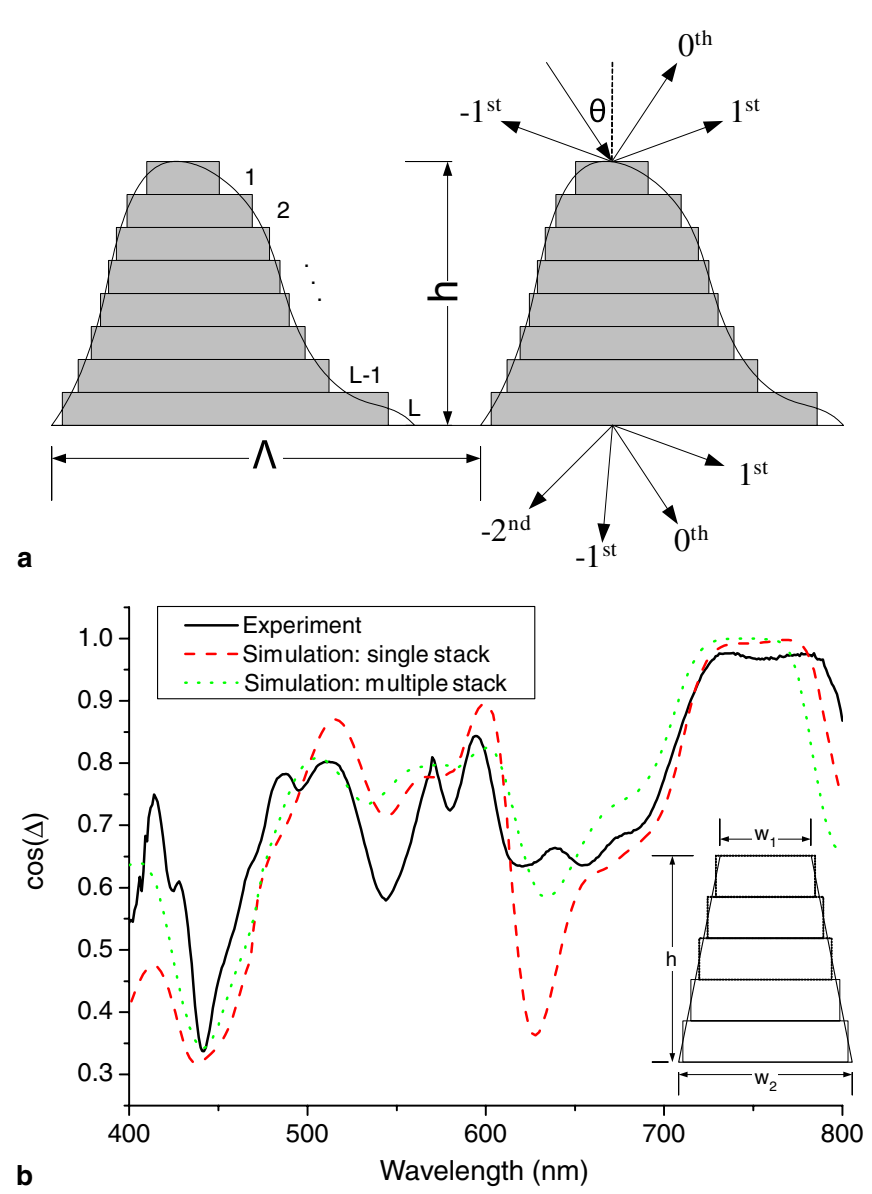

Fig. 7. (a) The periodic structure with an arbitrary profile can be approximated as a stack of lamellar gratings in RCWA. (b) Simulated spectra of the circular rod with a trapezoidal profile in its cross-section. The incident angle is $75^{\circ}$. The trapezoidal profile was approximated by five-layer 2D grating with an equal thickness.

unknown parameters to be determined and it will take long computational time to search for the optimal profile. A practical way is to generate a compete database as a library before the measurement.

The photonic band gap that forbids propagation of a certain frequency range of light is an important feature of the photonic crystal. As shown in Fig. 8(a), the band diagram and photonic bandgaps in TE-polarization (electrical filed parallel to the rods) for 2D square lattice was calculated by the plane-wave expansion method. Fig. 8(b) shows the corresponding reflectance spectrum in the $\Gamma-X$ direction calculated by RCWA method to confirm the photonic bandgaps directly. Ten lattice layers were considered and the rod height is assumed to be infinite in the simulation. From the two figures, there is a good agreement in the photonic bandgaps. The smaller peaks in the visible wavelength regime are due to the strong absorption of silicon in that regime.

\section{Conclusion}

In this work, RCWA was applied to analyze the measured ellipsometric parameters of the 2D PC slab. By

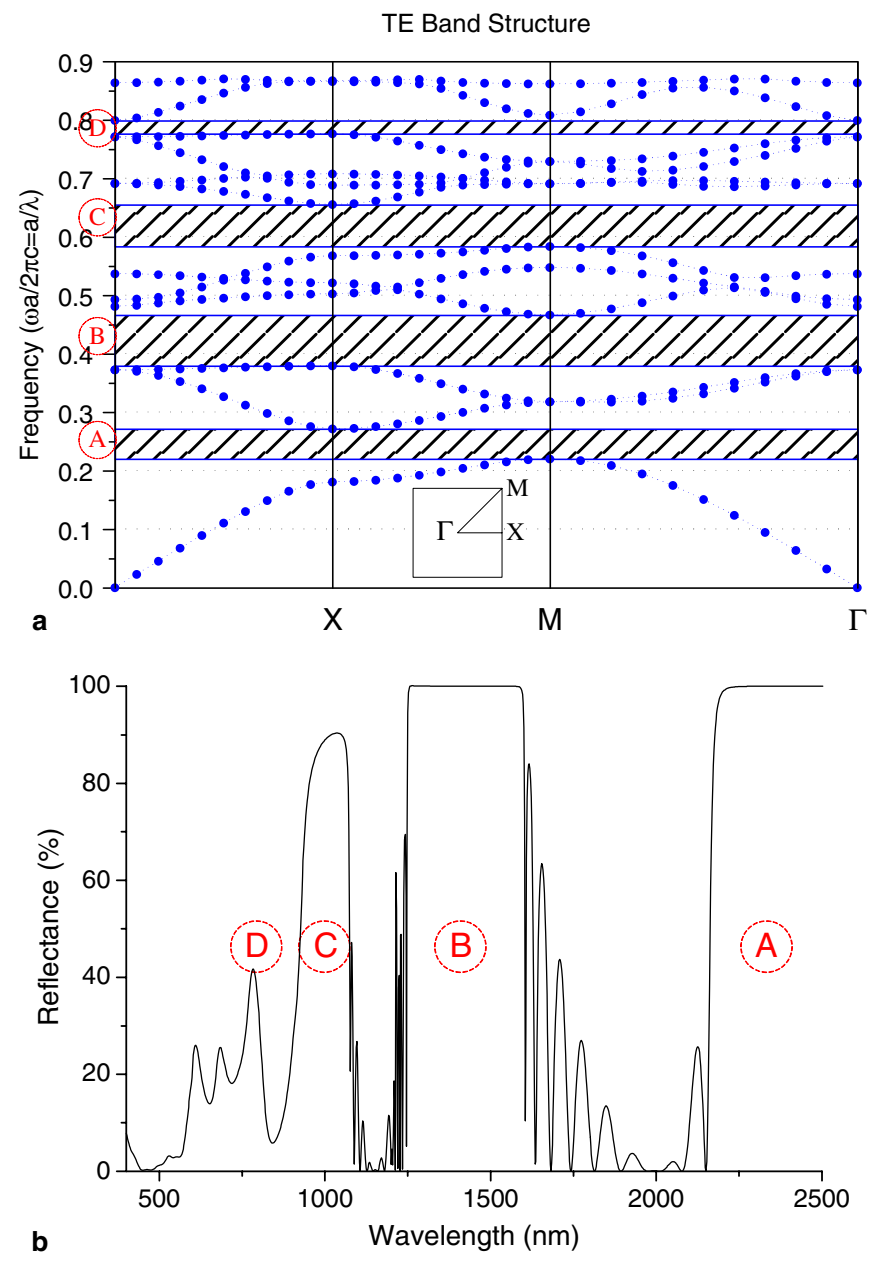

Fig. 8. (a) Band diagram and photonic bandgaps in TE-polarization for 2D square lattice. (b) The corresponding reflectance spectrum in the $\Gamma-X$ direction calculated by RCWA. The rod width is $396 \mathrm{~nm}$ and the rod height is assumed to be infinite in the simulation.

applying the symmetric condition of $2 \mathrm{D}$ grating in the RCWA algorithm, the computational efficiency can be increased up to around 6 times as compared with the conventional algorithm. When applying RCWA multi-layer approximation for the circular silicon rod with a trapezoidal profile in its cross-section, there is a good agreement between the simulated and measured ellipsometric spectra. The phase term, $\cos (\Delta)$, was found to be a better ellipsometric parameter as used to characterize the PC structure. The reflectance spectrum of the characterized 2D PC was also simulated by RCWA to reflect its photonic bandgap behavior directly. The simulated reflectance spectrum confirms with the bandgap diagram calculated by the planewave expansion method.

\section{Acknowledgments}

This work was supported by National Science Council, Taiwan, ROC (Grant No. 94A0501). The authors are grateful to the National Center for High-performance Computing for the support of the computing facilities. 


\section{References}

[1] E. Yablonovitch, Phys. Rev. Lett. 58 (1987) 2059.

[2] A. Scherer, O. Painter, J. Vuckovic, M. Loncar, T. Yoshie, IEEE Trans. Nanotech. 1 (2002) 4.

[3] J.S. Foresi, P.R. Villeneuve, J. Ferrera, E.R. Thoen, G. Steinmeyer, S. Fan, J.D. Joannopoulos, L.C. Kimerling, H.I. Smith, E.P. Ippen, Nature 390 (1997) 143.
[4] C.I. Hsien, H.L. Chen, W.C. Chao, F.H. Ko, Microelectron. Eng. $73-$ 74 (2004) 920.

[5] M.G. Moharam, E.B. Grann, D.A. Pommet, J. Opt. Soc. Am. A 12 (1995) 1068

[6] P. Lalanne, J. Opt. Soc. Am. A 14 (1997) 1592.

[7] E.N. Glytsis, J. Opt. Soc. Am. A 19 (2002) 702.

[8] C. Zhou, L. Li, J. Opt. A 6 (2004) 43. 